\title{
From Reputation Models and Systems to Reputation Ontologies
}

\author{
Rehab Alnemr and Christoph Meinel \\ Hasso Plattner Institute, Potsdam University, Germany \\ rehab.alnemr@hpi.uni-potsdam.de
}

\begin{abstract}
Reputation has been explored in diverse disciplines such as artificial intelligence, electronic commerce, peer-to-peer network, and multi-agent systems. Recently it has been a vital component for ensuring trust in web services and service oriented architectures domains. Although there are several studies on reputation systems as well as reputation models, there is no study that covers reputation ontologies especially the ones implemented using standardized frameworks like semantic technologies. In this paper, we show the evolution towards reputation ontologies and investigate existing ones in the domains of multi-agent systems, web services, and online markets. We define the requirements for developing a reputation ontology and use them to analyze some of the existing ontologies. The components and functionalities of reputation models and systems are described briefly and the importance of developing and using reputation ontologies is highlighted within the emergence of the Semantic Web and Semantic Web services.
\end{abstract}

Keywords: Reputation, Reputation Models and Systems, Ontology, Reputation Ontologies, Semantic Web.

\section{Introduction}

Reputation is a social control artifact that has been studied in psychology, sociology, economics, and computer systems. Reputation systems are used as a way of establishing trust between unrelated parties, especially if enforcement methods like institutional policies are not implemented. They may help lower the risks of online interactions, increasing the robustness and efficiency of internet-based applications. A reputation model describes all of the reputation statements, events, and processes for a particular context. This context is the relevant category for a specific reputation. The way such systems query, collect, and represent reputation varies. Some systems use stars or scaling bars as the visual format of reputation while others use numbers and percentages such as rating an e-market participant. Online reputation systems are the biggest and most obvious examples of these systems. It can be categorized based on the common features and properties of the web communities such as e-markets, activity sharing, social and entertainment sites, news sites, P2P systems and systems build upon the Semantic Web. [6] In service-oriented systems, quality attributes and ratings given by other services or service consumers are used to represent service reputation.

I. Wakeman et al. (Eds.): IFIPTM 2011, IFIP AICT 358, pp. 98-116. 2011.

(C) IFIP International Federation for Information Processing 2011 
There are extensive studies about reputation systems that discuss not only the current commercial ones but also proposed approaches from academia. For example, studies done by Jøsang in [18 and Sabater in 34 provide an exhaustive view of the status of the reputation community. However, there is still a confusion on the definitions and use of the associated terminologies between researchers and developers. Some describe their work to be a reputation system or a framework, others describe it as a model or a mechanism and few as an ontology. Moreover, there is still no explicit and standard theory of the cognitive components and processes which reputation is made of, despite the rapid advances in cooperation networks studies. According to [18, several algorithms for computing reputation were proposed in the past decade. However, commercial applications implementing trust and reputation mechanisms use relatively simple schemes than those proposed by research papers. In this paper, first we distinguish between the used terminologies then we focus on analyzing existing and proposed reputation ontologies and briefly show the difference between reputation mechanisms, models, frameworks, systems on one hand and ontologies on the other hand.

Lack of common terminology, proper definition, and means to exchange understandable reputation information, led to attempts to define reputation ontologies that can be used across several domains. In information systems, ontologies promote and facilitate interoperability as well as intelligent processing. They are developed to enhance knowledge reuse by sharing a common understanding of a domain that can be communicated between people, and heterogeneous and widely spread application systems. Usually, they are composed of a set of terms representing concepts (hierarchically organized) and some specification of their meaning. In this paper, we study the importance of developing such ontologies for reputation systems. We investigate existing reputation ontologies developed in multi-agent systems (MAS), web services and service-oriented environments (SOA), and online markets. Using a methodology for developing proper ontology, we define a set of questions that is later used in the analysis of these ontologies. The paper is organized as follows: in section 2 we briefly describe reputation systems and models and distinguish between the used terms. Section 3 explains the importance of ontologies followed by the requirements to develop one. In section 4 we investigate current reputation ontologies. Based on the requirements, we analyze these ontologies in section 5.

\section{Reputation Models, Frameworks, and Systems}

Since there is a vast literature showing reputation systems from different perspectives (i.e. 24], 18], threats in [1], mechanisms in [13]), here we will briefly point out several definitions to distinguish between some terminologies. Resnick 30] defines a reputation system as: "a system that collects, distributes, and aggregates feedback about participants' past behavior". It must have three properties to operate: long lived entities with an expectation for future interactions, ratings that can be captured and distributed, and past interactions' ratings used to guide 
the decision making. Conte and Paolucci [29] defines reputation-based systems as: "a spontaneous and implicit norm-based system for social control". Jøsang 18 defines reputation architecture as a network architecture which determines how ratings and reputation scores are communicated between participants in a reputation system. He identifies two main types for the networks:centralized and distributed architectures. Both architectures have a reputation computation engine, but each has a different communication protocol (i.e. centralized protocol for centralized architecture). A reputation computation engine computes the reputation value based on plenty of factors (according to the model used) such as one's own experience, others referrals, a combination of both, etc. Some of the used algorithms or computation functions are: summation, average [31, bayesian systems [23] 39], discrete trust models [2] 9, , belief models [17] 40], fuzzy models 33 36, cognitive as 14, etc.. A reputation model describes all of the reputation statements (i.e. a source rating a target), events, and processes for a particular context. They were developed using different approaches and different semantics. A reputation context is the relevant category for a specific reputation. A reputation system should describe therefore:

- Computation functions/mechanisms i.e. how to calculate reputation?

- Communication model i.e. how to collect and disseminate reputation?

- Participants i.e. who use and/or is affected by reputation?

- Resources i.e. what is the information used to calculate reputation?

- Representation model i.e. how to represent, view, or visualize reputation?

- Storage i.e. where and how reputation is stored?

- Functionalities and applications i.e. what are the benefits of using reputation in the domain of its creation

It should also describe how these components are integrated into a given system. Here, we distinguish between: reputation ontology, reputation system, model, or framework, reputation engine or mechanism, and reputation architecture. A reputation ontology describes the notion of reputation and the relations to the concepts that compose it, while a reputation system, model, or framework describes the collection, distribution, and aggregation of reputation information. A reputation computation engine or mechanism is one of the modules in a reputation system which shows how reputation value(s) are calculated. A reputation architecture is a set of protocols that determines how reputation values are communicated between the participants in a reputation system.

\section{Why Ontologies}

In [19], an ontology is defined as: "A set of terms of interest in a particular information domain and the relationship among them". Ontologies describe domaindependent as well as domain-independent knowledge. An Ontology defines common vocabulary for researchers who need to share information in a domain. In [22, the authors explain the reasons why anyone wants to develop an ontology. Some of them are [22]: 
- sharing common understanding of the structure of information

- enabling information reuse

- making domain assumptions explicit and clear

- separating domain knowledge from operational knowledge

- analyzing the domain knowledge

- ability to integrate existing ontologies describing portions of the large domain

Within the same community, ontologies enable mutual understanding among peers by providing precise semantics to concepts and relationships between these concepts. Our domain of focus in this case is reputation. According to Jøsang in [18, most reputation systems proposals from the academic communities lack coherence and are usually designed from scratch. Mostly, researchers do not build upon other researchers work. Usually because these proposals do not clearly define the involved concepts (i.e. reputation) and the knowledge in a standardized or formal manner. The lack of semantics of the concepts as well as of the elements included in reputation systems prevent others from reusing or extending -sometimes even understanding- the proposed work.

In order to define a standard notion of reputation, a general reputation ontology must be developed. However, it is important to separate, for instance, what is a reputation computation function from how it is computed. The former is domain knowledge while the latter is operational knowledge. Therefore, formalizing reputation concepts into ontologies has several advantages such as:

- creates a common understanding for reputation

- specifies the factors involved in computing reputation and the semantics of these factors

- separates the definition of reputation from how it is calculated

- enables the mapping between reputation concepts, in the current variety of reputation models

- facilitates the use of existing mapping and integration techniques in information systems for reusing reputation information

- increases the possibility of reputation interoperability and cross community sharing of reputation information

The semantic characteristics of reputation values are essential so that the participants are able to interpret them. These characteristics may differ from one domain/discipline to the other but the general description- as factors affecting the notion of reputation- is the same. For example, "service availability" is a factor that affects a web service provider's reputation in SOA while an agent's "trustworthiness" in MAS is a factor in calculating his rating for another agent. Both can be considered as quality attributes or reputation contexts.

The authors in 27] describe an ontology life cycle as: specification (why building an ontology), conceptualization (describing a conceptual model of the ontology), formalization (transform into formal model), implementation (implement in knowledge representation language), maintenance (update and correct), knowledge acquisition (of the subject by using elicitation techniques), evaluation (by judging technically the quality of the ontology), and documentation (report 
what was done, how, and why). Creating a general ontology that describes reputation involves separating clearly the closely related social artifacts like image 29] and reputation. In this paper, we discuss work that attempts to define and separate between reputation and trust concepts as well as analyzing those few ontologies that reached the implementation phase (not necessarily going through the preceding steps of conceptualization and formalization).

\subsection{Ontology Standards}

Developing such interoperable reputation ontologies requires a technology that can provide means of integrating data sources and methods to relate the data to its semantics. Semantic Web technologies were developed with the goal of providing common data representation framework in order to facilitate the integration of multiple sources to draw new conclusions, increasing the utility of information by connecting it to its definitions and to its context, and providing more efficient information access and analysis [8]. The Semantic Web organization 1 considers ontologies as one of the pillars of the Semantic Web. To achieve the main goal of Semantic Web, shared ontologies have to be established, which specify the fundamental objects and relations important to particular communities. Since these ontologies describe a concept in a certain domain, domain experts are often the ones who craft them. The representation languages includes XML Schema, RDF Schema 2 , the Web Ontology Language (OWL) 3 , and the Web Services Modeling Language (WSML) 4 . The advantage of defining an ontology using these technologies are: focusing on the representation of the semantics of the information, expressiveness, information reuse, easy discovery, and integration of information. Further information can be found in the references.

\subsection{Ontology Requirements}

Following the methodology of Grüninger [16] for ontology development, a reputation ontology should describe: the notion, the relation between the involved concepts, what computation function used to calculate the reputation value, in what domain it was collected, and the context of the computed reputation. The methodology involves defining a set of competency questions in the process of ontology development. These questions act as requirements in the form of queries that an ontology should be able to answer. Based on analysis and learning from others' experience, we define the following informal competency questions in the context of using and processing reputation in several domains:

Q1. Reputation definition: can we clearly define the notion of reputation within the domain?

\footnotetext{
${ }^{1}$ http://semanticweb.org/

${ }^{2}$ RDF Schema: http://www.w3.org/TR/rdf-schema/

${ }^{3}$ OWL-2: http://www.w3.org/TR/owl2-overview/

${ }^{4}$ WSML Working group:http://www.wsmo.org/wsml/
} 
Q2. Reputation Identity: in a specific context, can we define entities that can have reputation? can we define reputation roles such as source, target, evaluator, etc.?

Q3. Reputation representation: in a specific context, is the reputation value represented in a single format? if so, is it enough to express its meaning? how reputation will be represented, communicated, and visualized if necessary?

Q4. Reputation statement: what constitute as a reputation statement? what information does a reputation transaction hold?

Q5. Reputation computation mechanism: in a specific context, is there a property that defines and describes the mechanism by which reputation is computed?

Q6. Reputation context: is reputation related to its context? is there a property that expresses the relation between a reputation value and the context of its creation? and for a given entity, can we combine its reputation in different contexts?

Q7. Reputation factors: in a specific domain, can we define and describe the factors affecting reputation? does the reputation of the source (reputee) affect the calculation of reputation?

Q8. Reputation dynamics and temporal effect: does reputation change through time? if so, can we reflect this in the ontology? are there properties that reflect the change in reputation values? for example, is there a time validity property to reputation? for every new transaction, is the new value timestamped?

Q9. Reputation history: for a given entity, can we maintain the history of reputation values that said entity owned?

Q10. Reputation expressiveness: can we define and describe the semantics of the involved factors, contexts, relations, and concepts? is there a way to define as well as communicate the semantics of a reputation context?

This set of questions is not complete, it is rather an attempt to guide our analysis and discussion of the existing ontologies. According to [16, they should be used to evaluate the ontological commitment that has been made and evaluate the expressiveness of the ontology.

\subsection{A Literature of Defining Reputation Concepts}

Developing an ontology does not have to be from scratch. On the contrary, it is preferable to use the existing literature -if not extending an existing ontologyto define reputation concepts, form competency questions, and reach a formal model that leads to proper implementation. In the literature, reputation is defined as an expectation about an entity's behavior based on information about or observations of its past behavior [2]. In the business world, Balmer [7] defines two characteristics for corporate reputation: it evolves through time and is based on what the organization has done and how it has behaved. It deals with the cause of a problem, offers solutions, sets processes in motion, and monitors progress towards these solutions. Reputation definition evolves through the introduction 
of more complicated models. For example, Paolucci and Conte in [29] introduce a cognitive theory that clearly distinguish between two concepts- image and reputation. The computation algorithm was defined in 32 as the Repage model. One of the major efforts that has been done to define a set of terms about reputation concepts can be seen in the European project eRep 5. Based on the model described by 29, the project explains in details the elements of social evaluations, the process of transmitting them, and agent decisions regarding reputation. The model distinguishs between two social artifacts that pertain to the evaluation of a target: image and reputation. Image is the the output of the evaluation process of other agents and assumed to be true by the agent who holds it. Reputation is the voice the agent is spreading which is not necessarily the truth. One should make use of the literature on the subject before re-defining the concepts or the terminologies when developing a reputation ontology. However, sometimes it is essential to clearly distinguish one's perspective of a concept than other's vantage point by creating different terminologies.

\section{Existing Approaches to Reputation Ontologies}

In this section we describe some of the existing reputation ontologies developed and/or implemented with standardization in mind. We show how these ontologies were used as well as their limitations. They are categorized based on the domain they were created for. Some of these ontologies were created to define not only reputation but the associated concepts that will affect it. Others like [12] defines a reputation ontology in terms of its computation mechanism; that is; they define reputation as the mechanism used to compute it. We chose to show the graphical representation for each ontology to better illustrate the relation between its classes and their taxonomy. Finally we discuss these ontologies as opposed to the previously mentioned competency questions.

\subsection{For Multi Agents Systems}

Functional ontology for reputation (FORe) [11. The goal of this ontology is to represent the knowledge about reputation in psychology and AI in a structured form. The Ontology follows the categorization of reputation in law; following the work of [37. It extends the concept in the legal world to the social norm with different penalty of rule violation. While the legal penalty is a legal punishment, in the social world the penalty is having a bad reputation. The authors define reputation as a social product (an opinion or agreement) and a social process (contains a flow of information and influence on the social network). They present a functional ontology of reputation that contains four categories: Reputative Knowledge (deals with the agent's reward or penalty and models the products as well as the processes involved in the reputation notion), Responsibility Knowledge (associates a cause, whether it is intentional or based

${ }^{5}$ eRep project: http://megatron.iiia.csic.es/eRep/?q=node/93 


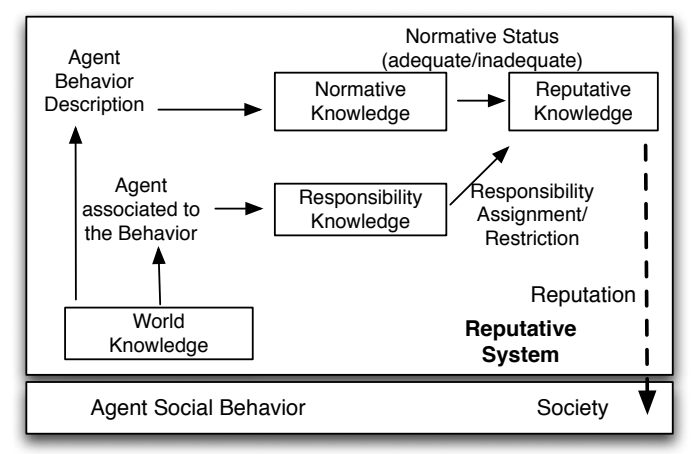

Fig. 1. Reputation Functional Ontology 11]

on circumstances, to a specific agent behavior), Normative Knowledge and World Knowledge (classifying the agent behavior and providing a model of social behavior). The distinction between these categories is based on the function that each component in the system performs. Since the ontology is complicated and there are plenty of classes involved, we show their detailed structure in figure 2 and the relation between the four categories in figure 1] Refer to [1] for the details of these components.

The authors in 38] used this ontology to examine different technologies in order to allow reputation interoperability between agents. This is done by asking the contributing agents to map their own ontology concepts to the functional ontology [11 and send the mapped information to the other agents that can in return convert it to their own model. The experiments were done using ART Testbed [15] which is a testbed to compare and evaluate between different heterogeneous reputation models or functions. They modified the testbed so that the mapping to a common ontology is no longer simple (which was a numerical value between 0 and 1 ) but rather sending interaction messages that are strings that hold queries and answers. The general architecture includes: the interaction module (receives a reputation message), mapping module (analyzes its contents), translated message (from the mapping module to the reasoning module) and the resulting query (the translated result sent to the interaction module). The work was extended in 25] to propose a separate mapping service that maps a reputation ontology to the functional ontology. The service was implemented so that agents do not have to map the ontologies themselves thus removing the mapping function from the agent architecture. Interoperability is achieved byt implementing two functions: mapping (assigning concepts and relations from one ontology to the other) and translating (application of the mapping function to translate sentences from one ontology to the other). Figure 3 illustrates the proposed service-oriented architecture (SOA) wherein mapping service was implemented as a web service using Protégé plugins.

Notes and comments. This ontology though gathering most of the notions associated with or relevant to reputation, do not provide a concrete way to represent 


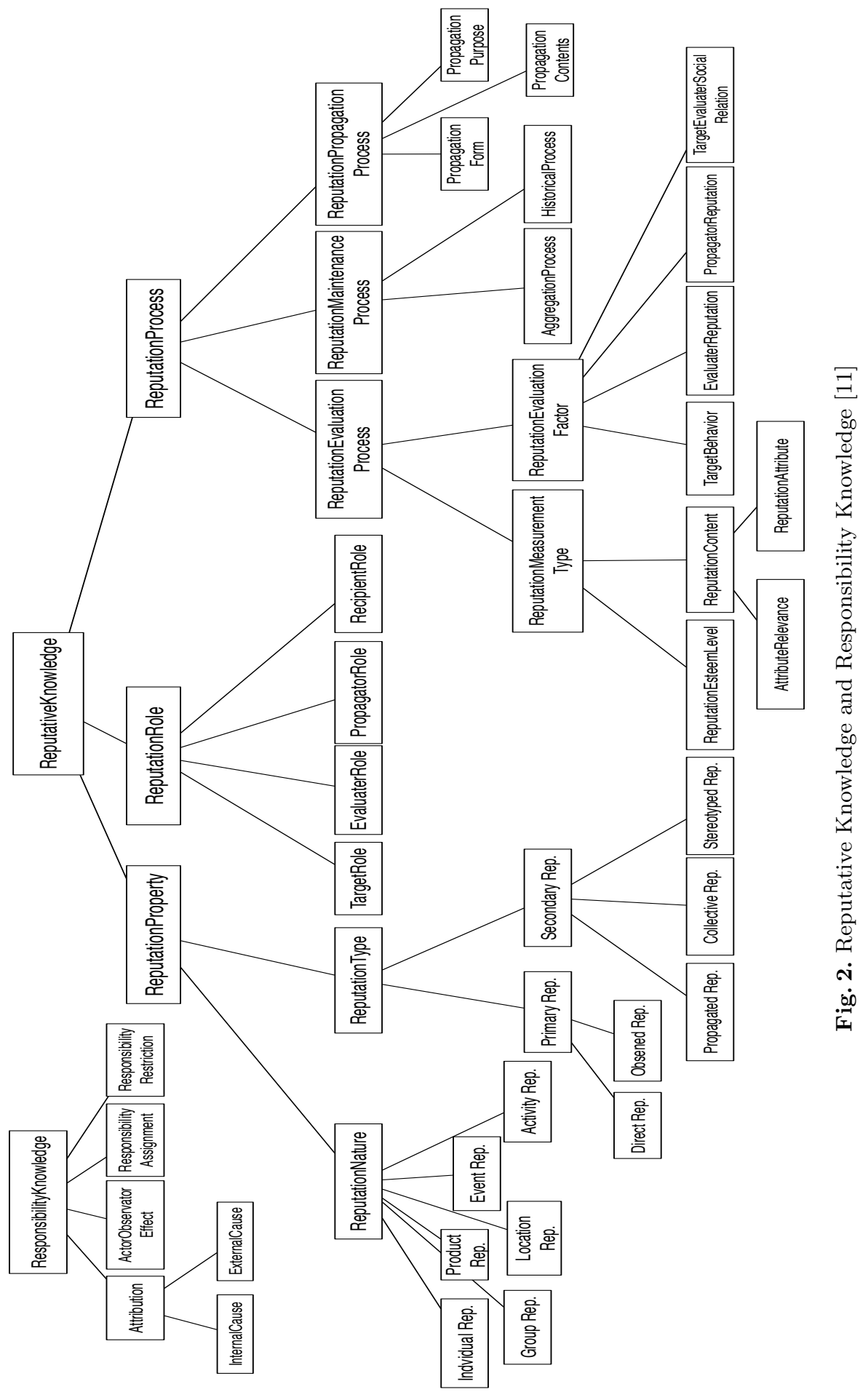




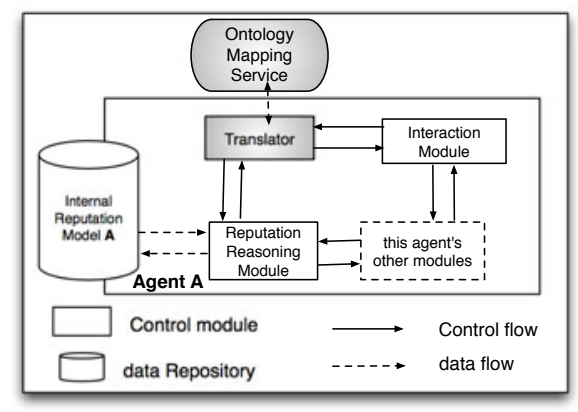

Fig. 3. SOARI Architecture 25]

and communicate this reputation; that is, a reputation representation. It is rather a meta-level description of the notions the surrounds reputation. Also, as illustrated in the figures, the functional ontology is complex and not all of its components are related to reputation transactions. Therefore, in order to adapt the approach used in 38 each agent must fully [understand] the ontology in order to: a] map his ontology- correctly- to it, b] answer any query sent from another agent. The ontology mapping service though beneficial but it still strictly requires that the involved reputation models are described using ontologies and specifically; OWL6. Moreover, the results showed that the binary mapping to FORe can not always be done and causes sometimes loss of information (e.g. mapping from Repage 32 to FORe, the concepts AgentReputation and AgentImage are mapped to ReputationNature concept in FORe).

Social evaluation and voice ontology [28]. Pinyol and Sabater use the concepts presented in the eRep project 7 and propose a common ontology in [28] for reputation (as a subset of the defined concepts in the project). They claim that the ontology allows the communication of social evaluations -reputation in this case- among agents using different reputation models. They argue that each agent use its own representation of the evaluations and interaction, therefore preventing communication between two agents using different reputation models. Even if the source knew which model the recipient is using, there is no reason to think that it will know the internal functionalities of other participants. They focus on the concepts concerning agents beliefs that deal with social evaluations and on the implementation with emphasis on the representation of the social evaluation values. The elements of the ontology are: an Entity is any object of the society being evaluated or having an active role in the evaluation (as Target, Source, Gossiper, Recipient), Focus is the context of the Evaluation, and Value describes the goodness or badness of this evaluation weighed by Strength to indicate how reliable the evaluation is. This measure of reliability is subjective and belongs to the interval $[0,1]$ with 1 being the maximum reliability and is

\footnotetext{
${ }^{6}$ OWL Web Ontology:http://www.w3.org/TR/owl-features/

7 eRep project: http://megatron.iiia.csic.es/eRep/?q=node/93
} 


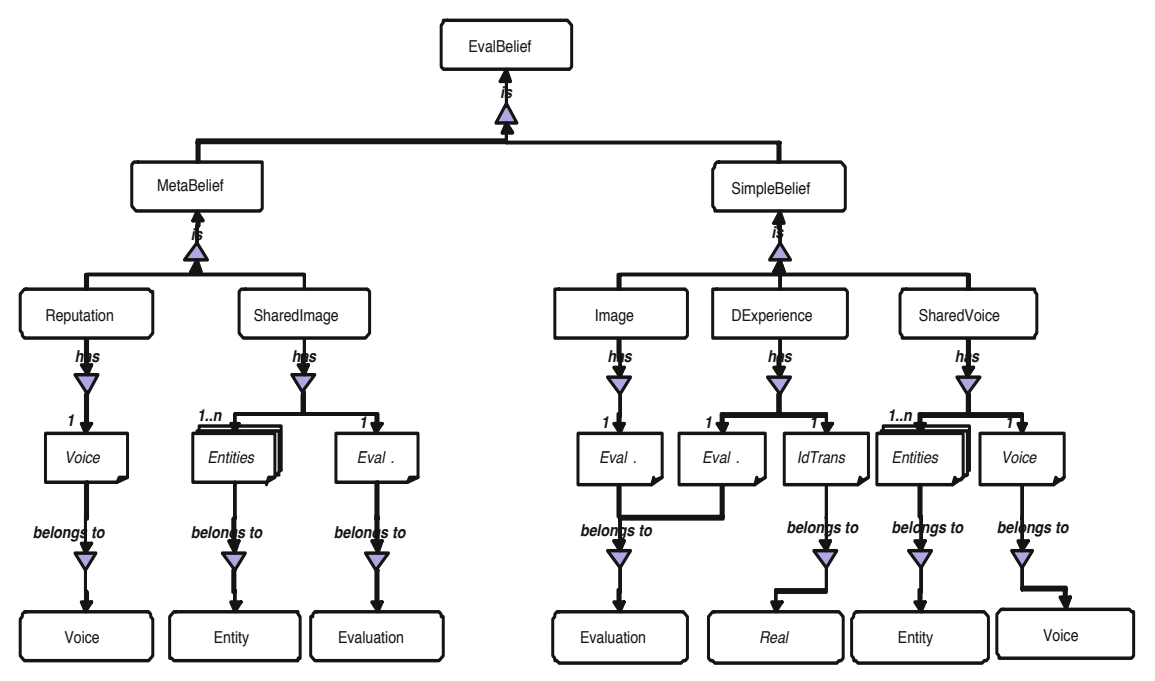

Fig. 4. Social evaluation and voice Ontology 28]

strongly related to an uncertainty factor called UncertaintyConversion (CU). It is the decision of an agent to use or ignore these factors. Evaluation encloses all the concepts involved in the social evaluation, while Voice is what actually being reported about the target's reputation. The ontology also presents a concept of evaluative belief EvalBelief which means what the agent holds in its belief system; SimpleBelief, a belief that the holding agent considers as true (that is not necessarily the voice being reported, hence, image belongs to this class), and MetaBelief, a belief about what others belief (the one being transmitted, hence, reputation belongs to this class). (See figure 4)

This work further discusses how the evaluation or these believes is represented because when developing a common ontology it is important to have a common understable representation of the evaluations. Therefore, the authors show a set of transformation functions between Boolean, Real, Discrete Set and Probability Distribution representations since they are the most common ones. They implemented an API interface of a set of common operations whose inputs and outputs are elements of the ontology, and must be implemented for each particular model. They show some implementation examples for famous models such as eBay 8 and Repage 32 by implementing its mapping to the ontology (only the first level of the API hierarchy of each model).

Notes and comments. The presented ontology though not as complicated as the functional ontology but also presents a level of complexity that can not be handled outside the multi-agent based community. However, it also covers all the factors affecting reputation and the process of decision making. Note also

8 eBay: http://www.ebay.com 
that it is a static ontology- a fact addressed by the authors- meaning that for every new reputation model a new API must be implemented. Dynamic ontology alignment is addressed by the ontology presented in 4.4 .

\subsection{For Web Services}

Service reputation ontology [21]. Maximilien and Singh presented a service reputation ontology in 21] to organize ratings (aggregated to reputation) to be used in service selection. They address the problem that trust criteria- that are involved in service selection- are not usually available in the service descriptions. The idea is that a service reputation is the aggregation of the ratings of the given service by other principals and is a vector of attribute values. The ontology includes domain independent as well as domain specific attributes and is described using DAML 9 (which formalizes models to express service capabilities through service descriptions).

In their architecture, the parties involved are called principals. Agencies are gathering and disseminating reputations and endorsements, and for each service used by the service consumer a proxy software agent is established. A ProxyAgent is responsible for: consulting outside registries and reputation and endorsement agencies, finding appropriate providers, recording feedbacks, learning from the experience, and finally sharing its knowledge. The ontology defines: a Service that has one Reputation which has one or more Ratings and is affected by History. A ReputationAlgorithm aggregates various attributes. A Principal rates a service and a RatingAlgorithm calculates the rating. Figure [5] illustrates the ontology and shows that a service reputation is a function of attributes that matter to a specific agent by adding a weight to the rating. This is the authors' interpretation of the real-world fact that reputation is subjective and depends on the domain of its creation. They also includes other factors affect service reputation such as attribute aggregation algorithm, the set of endorsers, and the damping factors for the ratings.

Notes and comments. So far, this ontology is the most expressive and practical ontology for service reputation. It is expressive because it captures most of the aspects that affect reputation and also because it addresses the effect of the domain on the attributes contributing to construct this reputation. Most of the systems addressing service reputation represent it as a single value such as [20] and [10] whereas in this ontology it is a vector of values. However, this vector of values does not have the semantics of the attribute embedded in the representation which makes it difficult for the destination (or the recipient of the transmitted reputation) to understand the source's interpretation of the attribute meaning.

\subsection{For e-Markets and Service-oriented Architecture}

e-Market and SOA ontology [12]. Chang et. al. describe an ontology for product, service, and agent reputation in the domain of e-markets. This was

${ }^{9}$ The DARPA Agent Markup Language (DAML): http://www.daml.org 


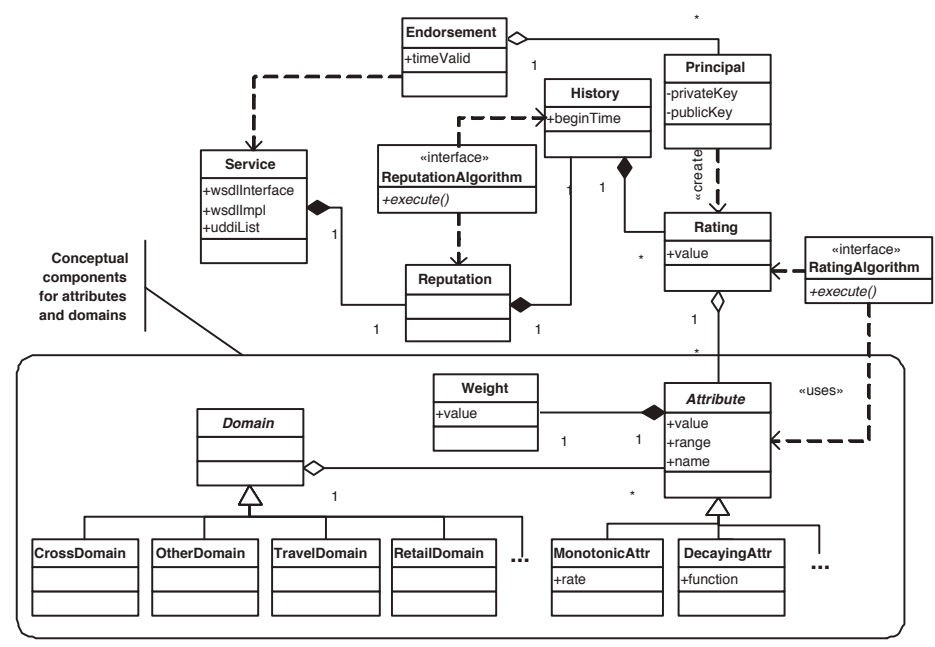

Fig. 5. Maximillian and Singh Service Reputation Ontology [21]

extended later to the domain of service-oriented architectures (SOA). They propose two definitions for reputation: a basic one and an advanced one. Basic Reputation ontology defines the reputation of a trusted agent as the aggregation of all the recommendations from all of the Third Party Recommendation Agents. Since this simple view do not address the dynamic nature of reputation (change over time) nor it ensures the depth of the context or the accuracy of the distribution, an advanced reputation was defined as the aggregation of all the recommendations from all of the Third Party Recommendation Agents weighed by the trustworthiness of the recommendation agents and the trustworthiness of the opinion. Figure 6 shows their ontology of advanced reputation and the following equations represent basic and advanced reputation ontologies respectively:

- Basic Reputation $=\cup($ RecommendationValue $)$

- Advanced Reputation $=\cup($ RecommendationValue $\times$ Trustworthiness $O f$ Opinion $\times$ Perceived $1^{\text {st }}, 2^{\text {nd }}$, and $3^{\text {rd }}$ opinion $\times$ TimeElapsedFactor $)$

Notes and comments. The presented ontology depends on the definition that reputation:"... developing the measure of trustworthiness from Third Party Agents recommendations, not by the Trusting Agents themselves" [12]. This greatly limits the view of reputation and the generic sense of reputation notion as well as over-simplifying its real nature. Hence, it is not generic enough to address several domains, especially an open one like SOA. Also, there is no definition of relevant concepts and ontology matching. Moreover, reputation at the end is a simple representation value which rather oversimplify the meaning of it.

\subsection{Generic Reputation Ontology}

Reputation Object ontology [5]. Most of the existing work on reputation systems focuses on improving the calculation of reputation values, preventing 


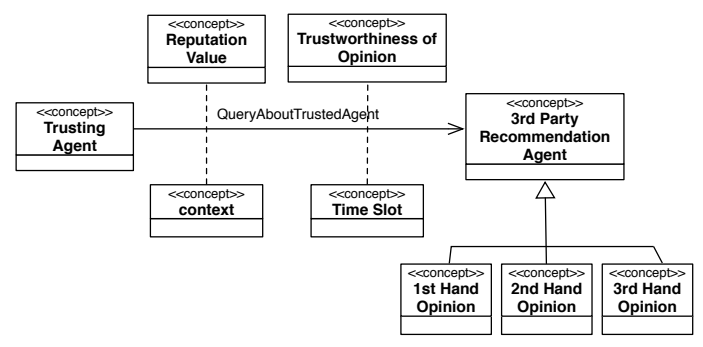

Fig. 6. Chang et. al. Reputation Ontology of the Trusted Agent 12 .

malicious actions, and the deployment into the business world where reputation is mostly represented in a singular value form. This work focuses on how to represent reputation to reflect its real-world concept (i.e. non-general, context specific, and dynamic). The argument is that in most reputation systems the context of a reputation value is not embedded within the given reputation information. Mostly because it has the single value format. Since reputation changes with time and is used within a context and every domain has its own information sources as well as its own requirements, the representation -not the calculation- of reputation should be unified between communities in order to facilitate knowledge exchange. In this ontology reputation is represented as a new form of reputation value: Reputation Object $(R O)$. This object holds information on the reputation of an entity in multiple contexts. The ontology's components are: a ReputationObject hasCriteria of one or multiple instances of class Criterion or QualityAttribute (for a service, the criterion describing service reputation is referred to as a quality attribute). The criterion is collected using a CollectingAlgorithm and hasValue ReputationValue. Each criterion instance has a ReputationValue (which includes the currentValue, its time stamp, and a simple list of its previous values called historyList) that in turn has the range of values defined in PossibleValues. It describes the data type that the criterion can have or a specific set of values (literals or resources URI) evaluating this criterion (e.g. a set of integers $\{1,2,3,4\}$ describing 4 trust levels or a set of Strings $\left\{\right.$ "good $^{\prime \prime}$, " bad", , excellent" $\}$ describing a user opinion). Each time a criterion is being evaluated (i.e. a new entry value for this criterion), a new currentValue is calculated using the ComputationAlgorithm which is the reputation computation function/engine used with this criterion such as sum, avg, etc..

Since it is not always easy to identify intuitively what the highest reputation value is - among the defined possible value set -, the PossibleValues class has an orderedList that is ordered from the relatively highest reputation value to

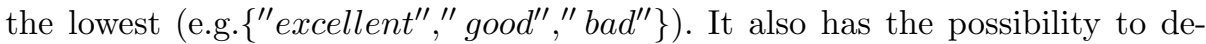
fine a comparison and ordering function; OrderFunction to compare between values within each criterion and to be used by the reasoning engine. A RO is constructed either offline or during negotiation process. It's a generic object that changes according to the domain and the user preference but in general it holds 


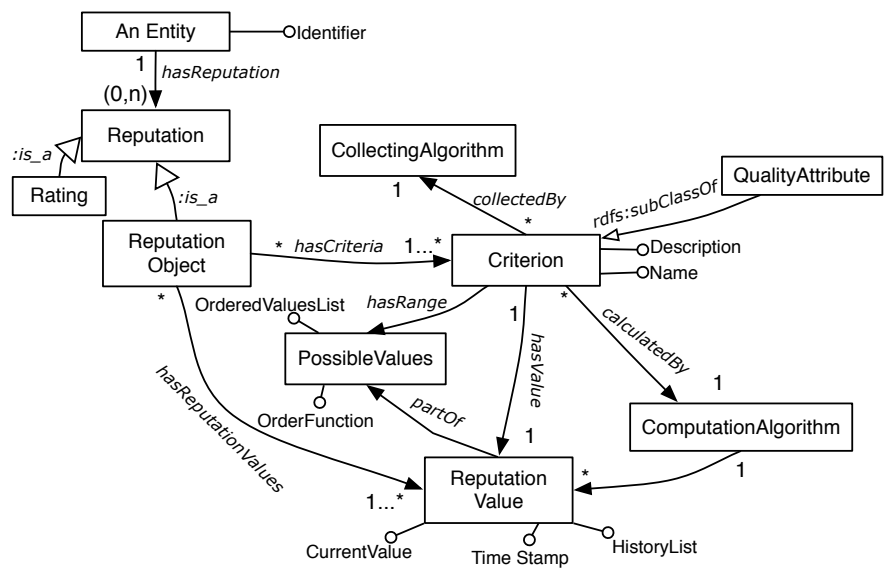

Fig. 7. Reputation Object Ontology [5]

a profile (functionality, quality, ratings, etc.) about an entity (service or agent) which is collected from heterogeneous information sources. The ontology is implemented using Protégé-OWL10. A java library was also developed to facilitate the integration of the ontology within any system on the implementation layer. The implementation for processing an RO was developed using Jena-API 11 . This ontology was used to represent an entity's reputation in several domains such as multi-agent based system (in 26], as the reputation of an agent and a way for decision making), for usage control in Internet-of-services (IoS) [4], and as an underlying ontology for a SOA reputation service in [3] that was later used in 35 for cloud service provider selection.

Notes and comments. This ontology was designed mainly to facilitate reputation information exchange or reputation interoperability in any domain. Using this ontology, a dynamic ontology alignment is possible between two entities since reputation information (that helps in the alignment specially during runtime) are embedded in the reputation object. However, since the ontology focuses on representation, it does not address factors like transformation functions. Though one can still use the functions presented in [28] (section 4.1) to enhance the use of the ontology.

\section{Discussion}

Despite that the aforementioned ontologies provide broad knowledge about reputation, most of them still represent reputation as a single value. Maximillian \&Sangh approach (section 4.2) perceives it as a single quality-attributes vector which increases the comprehensiveness of service reputation. The complexity

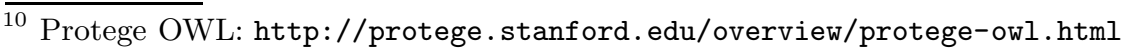

11 Jena framework: http://jena.sourceforge.net/
} 





of the functional ontology FORe (section 4.1) prevents it from being adapted despite of the presented mapping service in SOARI. While the ontology presented by Chang (section 4.3) reflects the existing approaches in online markets, it inherits their problems of over-simplifying the meaning of reputation, hence alienating factors that affect reputation calculation. The discussed ontologies are implemented using standardized technologies such as OWL and DAML-S. Pinyol \&Sabater present the elements of their ontology as an API interface which can be reused and the RO ontology provides a Java library and is described in OWL. Back to the competency questions in section 3.2, we find that some of these ontologies answer most of said questions as explained in table 1. Note that each one was created from a different point of view and for different purposes. However, when one intends to use an ontology, it is important to know its features.

\section{Conclusion and Future Wrok}

Reputation ontologies create a common understanding of the notion as well as facilitate reputation exchange. While there is a vast body of reputation systems studies, there is no study that covers reputation ontologies. In this paper we distinguish between reputation models, mechanisms, systems and reputation ontologies. We show why developing ontologies is important and construct a set of competency questions to guide the analysis of the existing reputation ontologies. Furthermore, some of these ontologies are described in this study and are analyzed based on the aforementioned competency questions. Until now, reputation systems do not use or even share one common ontology to facilitate reputation exchange. The initiative by the ORMS12 is trying to achieve that though it is still in its early stages. In order to use the resulting reputation ontology on a web scale, it should be compliant with current semantic web or web services standards and deployable on available Semantic Web infrastructure. It is encouraged to use standardized technologies to describe and exchange reputation information in a format that can be understandable to both humans and machines. This will greatly affect the decision making process in open environments such as service oriented architectures. We developed the ontology described in 4.4 with these goals in mind and we plan to continue to enhance the ontology as well as extending the competency questions to act as a guide reference for future ontology development or integration.

\section{References}

1. Reputation-based Systems: a security analysis (2007)

2. Abdul-Rahman, A., Hailes, S.: Supporting trust in virtual communities. In: Proceedings of the 33rd Hawaii International Conference on System Sciences, vol. 6 . IEEE Computer Society, Los Alamitos (2000)

12 Open Reputation Management Systems: http://www . oasis-open.org/committees/tc_home.php?wg_abbrev=orms 
3. Alnemr, R., Bross, J., Meinel, C.: Constructing a context-aware service-oriented reputation model using attention allocation points. In: Proceedings of the IEEE International Conference on Service Computing (2009)

4. Alnemr, R., König, S., Eymann, T., Meinel, C.: Enabling usage control through reputation objects: A discussion on e-commerce and the internet of services environments. Special issue of Trust and Trust Management, Journal of Theoretical and Applied Electronic Commerce Research (2010)

5. Alnemr, R., Paschke, A., Meinel, C.: Enabling reputation interoperability through semantic technologies. In: ACM International Conference on Semantic Systems. ACM, New York (2010)

6. Alnemr, R., Quasthoff, M., Meinel, C.: Taking Trust Management to the Next Level. Handbook of Research on P2P and Grid Systems for Service-Oriented Computing: Models, Methodologies and Applications

7. Balmer, J.M.T., Greyser, S.A. (eds.): Revealing the corporation. Routledge, New York (2003) ISBN:0415284201, http://gso.gbv.de/DB=2.1/ CMDACT=SRCHA\&SRT=YOP\&IKT=1016\&TRM=ppn+349820163\&sourceid=fbw_bibsonomy

8. Berners-Lee, T., Hendler, J., Lassila, O.: The semantic web. Scientific American Magazine (May 17, 2001)

9. Carbone, M., Nielsen, M., Sassone, V.: A formal model for trust in dynamic networks. In: Proc. of International Conference on Software Engineering and Formal Methods, pp. 54-63 (2003)

10. Cardoso, J., Sheth, A.P., Miller, J.A., Arnold, J., Kochut, K.: Quality of service for workflows and web service processes. J. Web Sem (2004)

11. Casare, S., Sichman, J.: Towards a functional ontology of reputation. In: Proceedings of the Fourth International Joint Conference on Autonomous Agents and Multiagent Systems, p. 505 (2005)

12. Chang, E., Hussain, F.K., Dillon, T.: Reputation ontology for reputation systems. In: Meersman, R., Tari, Z., Herrero, P. (eds.) OTM 2006 Workshops. LNCS, vol. 4278, pp. 1724-1733. Springer, Heidelberg (2006)

13. Dellarocas, C.: Reputation mechanisms (2005)

14. Esfandiari, B., Chandrasekharan, S.: On how agents make friends: Mechanisms for trust acquisition (2001)

15. Fullam, K.K., Klos, T.B., Muller, G., Sabater, J., Schlosser, A., Topol, Z., Barber, K.S., Rosenschein, J.S., Vercouter, L., Voss, M.: A specification of the agent reputation and trust (ART) testbed: Experimentation and competition for trust in agent societies. In: The 4th International Joint Conference on Autonomous Agents and Multiagent Systems (2005)

16. Grÿninger, M., Fox, M.S.: Methodology for the design and evaluation of ontologies. Basic Ontological Issues in Knowledge Sharing (1995)

17. Jøsang, A.: A logic for uncertain probabilities. Int. J. Uncertain. Fuzziness Knowl.Based Syst. 9, 279-311 (2001)

18. Jøsang, A., Ismail, R., Boyd, C.: A survey of trust and reputation systems for online service provision. Decision Support Systems, 618-644 (2007)

19. Kashyap, V., Bussler, C., Moran, M.: The Semantic Web, Semantics for Data and Services on the Web. Springer, Heidelberg (2008)

20. Liu, Y., Ngu, A.H., Zeng, L.Z.: Qos computation and policing in dynamic web service selection. In: Proceedings of the 13th international World Wide Web conference, pp. 66-73. ACM, New York (2004)

21. Maximilien, E.M., Singh, M.P.: An ontology for web service ratings and reputations (2003) 
22. McGuinness, D.L.: Ontologies come of age. In: Spinning the Semantic Web: Bringing the World Wide Web to Its Full Potential. MIT Press, Cambridge (2003)

23. Mui, L., Mohtashemi, M., Halberstadt, A.: A computational model of trust and reputation for e-businesses. In: Proceedings of the 35th Annual Hawaii International Conference on System Sciences, HICSS 2002. IEEE Computer Society, Los Alamitos (2002)

24. Mui, L., Halberstadt, A., Mohtashemi, M.: Evaluating reputation in multi-agents systems. In: Falcone, R., Barber, S.K., Korba, L., Singh, M.P. (eds.) AAMAS 2002. LNCS (LNAI), vol. 2631, pp. 123-137. Springer, Heidelberg (2003)

25. Nardin, L.G., Brandao, A.A.F., Sichman, J.S., Vercouter, L.: An ontology mapping service to support agent reputation models interoperability. In: 11th Workshop on Trust in Agent Societies, Estoril, Portugal (2008)

26. Paschke, A., Alnemr, R., Meinel, C.: The rule responder distributed reputation management system for the semantic web. In: RuleML-2010 Challenge, Washington DC, USA. ACM, New York (2010)

27. Pinto, H.S., Martins: Ontologies: How can they be built? Knowl. Inf. Syst. 6, 441-464 (2004)

28. Pinyol, I., Sabater-Mir, J., Cuni, G.: How to talk about reputation using a common ontology: From definition to implementation. In: Proceedings of the Ninth Workshop on Trust in Agent Societies, Hawaii, USA, pp. 90-101 (2007)

29. Conte, R., Paolucci, M.: Reputation in Artificial Societies. Social Beliefs for Social Order (2002)

30. Resnick, P., Kuwabara, K., Zeckhauser, R., Friedman, E.: Reputation systems. Commun. ACM, 45-48 (2000)

31. Resnick, P., Zeckhauser, R.: Trust among strangers in Internet transactions: Empirical analysis of eBay's reputation system. In: The Economics of the Internet and E-Commerce, pp. 127-157 (2002)

32. Sabater, J., Paolucci, M., Conte, R.: REPAGE: REPutation \& imAGE among limited autonomous partners. Artificial Societies \& Social Simulation Jour. (2006)

33. Sabater, J., Sierra, C.: Regret: reputation in gregarious societies. In: Proceedings of the Fifth International Conference on Autonomous Agents, AGENTS 2001, pp. 194-195. ACM, New York (2001)

34. Sabater, J., Sierra, C.: Review on computational trust and reputation models. Artif. Intell. Rev. 24, 33-60 (2005)

35. Schnjakin, M., Alnemr, R., Meinel, C.: A security and high-availability layer for cloud storage. In: The 2nd Int. Workshop on Cloud Information System Engineering (Springer CISE 2010) (2010)

36. Sen, S., Sajja, N.: Robustness of reputation-based trust: boolean case. In: Proceedings of the First International Joint Conference On Autonomous Agents and Multiagent Systems, AAMAS 2002, pp. 288-293. ACM, New York (2002)

37. Valente, A.: Legal Knowledge Engineering- A modeling Approach (1995)

38. Vercouter, L., Casare, S.J., Sichman, J.S., Brandão, A.A.F.: An experience on reputation models interoperability based on a functional ontology. In: Proceedings of the 20th International Joint Conference on Artifical Intelligence, San Francisco, CA, USA, pp. 617-622 (2007)

39. Whitby, A., Jøsang, A., Indulska, J.: Filtering out unfair ratings in bayesian reputation systems (2004)

40. Yu, B., Singh, M.P.: An evidential model of distributed reputation management. In: Proceedings of the First International Joint Conference on Autonomous Agents and Multiagent Systems, AAMAS 2002, pp. 294-301. ACM, USA (2002) 